\title{
APPLICATION OF ANALYTIC HIERARCHY PROCESS FOR FACULTY SELECTION AT NEPALESE UNIVERSITIES
}

\begin{abstract}
In recent times, with the increasing number of both educational institutions and the students, Nepalese education sector has become competitive. Institutions are working very hard to get qualified students as well as qualified faculties. Most of the institutions have developed competitive entrance examination system to select good students. But the selection of faculties becomes a difficult process as multiple criteria and sub-criteria need to be considered. Further, the process becomes more complex if we consider the perspective of two most important actors: the management and the students. It is a case of Multi Criteria Decision Making and AHP has already proven to be a useful tool in such scenarios. In this study, various factors, sub-factors associated with faculty selection have been identified and AHP has been used to prioritize these factors and sub-factors. The analysis has been done from the perspective of management, peer faculties and students. The research aims to provide a guideline for the faculty recruitment team at the universities regarding employee selection and can be applied to other educational institutions as well.
\end{abstract}

Keywords: AHP, MCDM, faculty selection.

\section{Introduction}

All the productive sectors in the world are getting competitive and education sector is not an exception. Most of the educational institutions are focusing on differentiating their services so that they can separate their services from others and can provide competitive advantage. There is no single solution valid for all the institutions as what works for one may not work for the other. It has been observed that the institutions are trying to focus on long term sustainability with the adaptation of strategic planning. Two of the key areas that have been identified are recruitment and selection of best faculties and enrollment of best students. In case of students' enrollment, most of the Nepalese educational institutions have developed a criterion through competitive entrance examination entrance system but they do not have proper framework for the selection of most suitable faculties. The faculty selection procedure they have been using need to be revised as there has been huge change in the education system and market dynamics which needs to be addressed by the institutions if they want to be competitive.

It has been evident that faculties for the modern education system need to be equipped with several expertises apart from academic excellence. One has to consider several criteria and sub-criteria. This is a true case of multi criteria decision making (MCDM) where multiple conflicting issues need to be considered. Among various tools available for MCDM, Analytic Hierarchy Process (AHP) has been adopted in this research. 


\section{Motivation for the research}

In the last decade, Nepalese higher education sector has seen a continuous increase in competition. The increasing trend of Nepalese students going abroad for higher education has further intensified the competition. Apart from inadequate facilities, inappropriate faculties occupying the faculty position is also among the important reasons for Nepalese students preferring developed countries to Nepal for their higher education. Development of infrastructure require huge money which requires long time for the economically weak Nepalese institutions but recruiting best suitable faculty can be done by developing proper guideline and a procedure in selection process. Further, it becomes very important for the institute to identify the most important criteria and sub-criteria which should be emphasized to achieve educational excellence and stand ahead in competition.

\section{Problem statement}

Most of the universities have faculty recruitment plan and procedure but mainly those are concentrated on academic achievement of the candidates. It has been evident that the policy they have adopted for several years needs amendment as it cannot address the modern education system. Further, due to limitation in economy it is difficult to make improvement in other areas like excellence in laboratory and other infrastructure. They need to bring out excellence and competitive advantage with minimum cost. To achieve this they need to select most appropriate faculties which can provide core competency in their business. It is important to develop a path that can guide management to select the best person that can make the institution competitive. This study intends to seek answer to the following research questions.

$>$ What should be the faculty selection path for Nepalese universities to achieve academic excellence?

$>$ Which is the most important factor to be considered while selecting a university faculty in Nepal?

\section{Literature Review}

Several researchers have highlighted faculty selection as an important step for the sustainability of an organization. The major criteria highlighted in these studies were: education, experience, scholarly activities, technological skills, flexibility in teaching capabilities, friendly conduct and experience working with diverse populations (Frangos, 2014 and Grandzol, J. R. 2005). Stöcklin S. (2010) stated some required dimensions for teachers as: knowledge of the subject matter, pedagogical knowledge with regard to the manner in which subject matter should be taught, the ability to assess student learning, fluent communication skills, and an ability to foster student's responsibility for their own learning. Oxford University Department of Education (2018) has listed major criteria for interview process as: academic, personal attributes, willingness and potential to learn, professional values and practices and school focus issues. Dočkalíková, I. and Kashi, K. (2013) used AHP in medium sized organization for employee selection. The criteria used were: Education and practice, Knowledge and skills and Personality requirements. AHP is capable of breaking complex problems into smaller parts that can be easily handled by human intelligence (Saaty, 2008)

\section{Objectives}

The major objective of this research is to develop a decision making model for faculty selection in universities and colleges. 


\section{Research Design}

This research adopted four tools in addition to literatures: expert opinion, interviews, questionnaire, and model development using AHP. A pair wise comparison questionnaire based on AHP model has been used for data collection. In total 40 valid samples were collected.

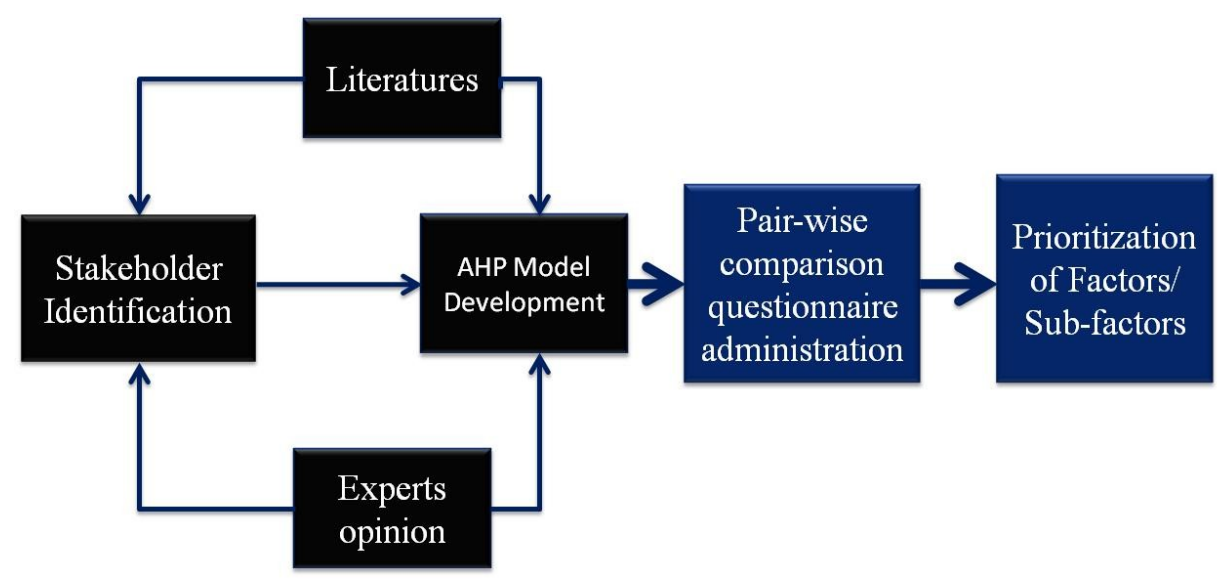

Figure: Research Design of University Faculty Selection

\section{Model Development}

The model developed during the process consists of four levels. Goal: Selection of a university faculty is at level one followed by major actors at level two. Level three and level four consists of factors and sub-factors respectively.

\section{Selection of a University Faculty}

\section{Students}
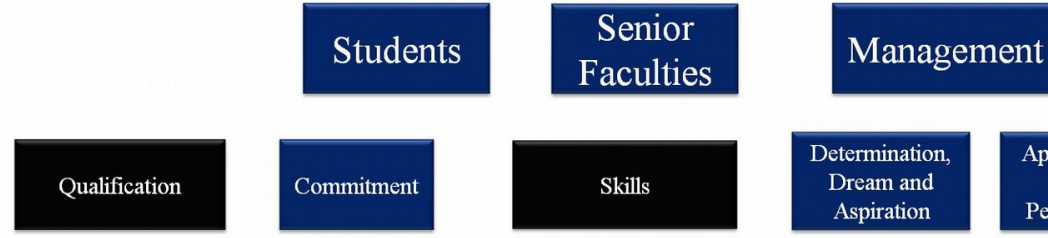

$$
\begin{gathered}
\text { Level } \\
2
\end{gathered}
$$

HS: Hard Skills, CS: Computer Skills, TS: Teaching Skills, ES: English Skills, SS: Soft Skills

Figure: AHP Hierarchical Model

\section{Limitations}

The method relies on subjective judgments for the analysis. The weights that are assigned to the decision makers can be tilted in favor such that the result will reflect the opinion of a selected few people. Maintaining consistency is also a very critical factor for the members' views to be accurately reflected in the results. Within a small group, an inconsistent input from a single member can immediately have an effect on the overall rankings. 


\section{Conclusions}

This is the first ever research done in Nepalese universities for faculty selection from the perspective of MCDM. The research can be a guideline for management in selecting appropriate faculty for the university. Although the research has been done in Nepalese context and at the higher education but it can be applicable in other countries universities as well as in schools.

\section{Key References}

Frangos, C. C. et al (2014), Student Preferences of Teachers and Course Importance Using the Analytic Hierarchy Process Model, Proceedings of the World Congress on Engineering Vol II, WCE, London, U.K., July 2 - 4

Grandzol, J. R. (2005), Improving the Faculty Selection Process in Higher Education: A Case for the Analytic Hierarchy Process, Association for Institutional Research, 6, $1-13$

Dočkalíková, I. and Kashi, K. (2013), Employees` Recruitment: Selecting The Best Candidates By The Utilization Of AHP And WSA Method, The 7th International Days of Statistics and Economics, Prague, September 19-21.

Saaty, T. L. (2008), Decision making with the analytic hierarchy process, Int. J. Services Sciences, 1(1), 83-98.

Stöcklin, S. (2010). The Initial Stage of a School's Capacity Building, Educational Management Administration \& Leadership 38(4), 443-453 